We have followed thus half a dozen typical cases of hereditary hæmophilia, in which hæmorrhages were occurring every month or two months; after treatment they ceased for several years to show any symptoms of hæmophilia. At the outset their blood required several hour's for coagulation, but at the end only 45,30 , or 14 minutes. The complaint had aged in several months as much as it would have done in 20 or 30 years without assistance.

The prognosis is transformed by the continuous serum treatment. 'Whilst Carrière, summing up a mass of hæmophiliac cases, estimates that 89 per cent. of patients do not live beyond 20 years, I have not lost one out of more that 50 patients. Moreover, under prolonged treatment the complaint becomes so attenuated that it seems as if treatment might even be given up after several years; this was well shown in one of our patients who, after six years of continuous treatment, went through five years of war without misadventure.

The important question presents itself whether, in women of a hæmophiliac stock, preventive serum treatment during pregnancy would enable them to bear healthy children. We have not yet had any opportunity to make any experiments in this direction.

Technique of Preventive Treatment.

The technique consists in making subcutaneous injections of blood serum in doses of $20 \mathrm{c.cm}$. every two months. Later on, after considerable improvement, injections are only necessary every three months. It is hardly possible to put off the injections any further.

In this continuous cure we have generally used animal serums without reason to regret it; but, if it could be done easily, human serums would be preferable. Chalier (of Lyons) has successfully used maternal serum, injected in doses of 30 to $40 \mathrm{c.cm}$. every fortnight.

Very good results have been obtained in hæmophiliac accidents by transfusion of blood. This method, though legitimate in serious hæmorrhage, cannot be recommended as habitual treatment, since blood tranfusions cannot be renewed every two months for years on end. In any case, we would utter a warning against subcutaneous hæmotherapy. In a serious case we have seen, after a dose of $25 \mathrm{c} . \mathrm{cm}$. of human blood, an enormous hæmatoma form in the back and abdominal wall, the clot acting as a foreign body and causing a copious hæmorrhage.

\section{COAGULATION OF THE BLOOD:}

A STUDY OF THE ANTI-COAGULANT ACTION OF SODIUM CITRATE AND OF THE PART PLAYED BY CALCIUM IN THE BLOOD.

BY MARCEL BLOCH,

CIEF DE LABORATOIRE IN THE FACCLTY OF MEDICINA, PARIS,

THE study of the coagulation of the blood, which ought to be of great use in nosography, has been hampered by the insufficiency of the methods to measure its pathological variations. It is a phenomenon which lends itself with the utmost difficulty to exact measurements. In another place ${ }^{1} \mathrm{I}$ described numerous methods based on the speed of coagulation in vitro (methods of Vierordt, De Mutach, Biff, Schultz, Angelo Petrone, Brodie and Russell, Wright, and Sabrazès) MM. Achard and Binet have just described a neat technique. The methods of Hayem and P.E. Weil are the simplest, and these workers have made a profound study of blood coagulation in numerous pathological states.

Retardation of coagulation in vitro can be observed in normal subjects and in affections such as pneumonia, acute articular rheumatism, associated with an excess of fibrin in the blood; but though direct examination may be able to prove states of diminished coagulability, it is only with difficulty that it allows of appreciating states of exaggerated coagulability. Finally, it is very hard to avoid causes of error.
Consequently the speed of coagulation in vitro does not always accurately represent the coagulability. To find some method of measuring "the forces producing coagulation" as they exist in the blood in the vessels it is necessary to prevent them from determining coagulation in vitro, to immobilise them whilst leaving them intact, and to restore their activity so as to measure them in the laboratory. ${ }^{2}$ The use of citrate of sodium has enabled me to fulfil these conditions.

Action of Chemical Anti-coagulants in Vitro.

Very many substances are capable of hindering blood coagulation in vitro (acids, alkalis, salts, extracts of organisms, venoms, alcohol, \&c.). But those alone of importance in establishing a method of measuring are such as are strictly measurable, and do not a priori seriously affect the important elements of the blood, as do acids, alcohol, \&c. The most carefuliy studied and frequently used anti-coagulating salts are the oxalates of sodium and potassium, fluoride, and citrate of sodium.

The action of the oxalates is well known from the work of Arthus and Pagès. ${ }^{3}$ A proportion of about 1 per 1000 in the blood causes incoagulability by the precipitation of calcium in the form of an insoluble compound. The introduction of a new soluble calcium compound (even solubilised calcium oxalate) into the oxalate mixture restores the coagulating property. Theoretically, it is of interest to add that Hammarsten and Pekelharing have shown that the process of coagulation in the oxalated blood is arrested at the stage of the formation of the fibrin-ferment or thrombin. The calcium salts would therefore be necessary to the transformation of the prothrombin into thrombin. But as soon as the thrombin is formed the calcium salts are no longer necessary, and coagulation proceeds even in their absence.

Sodium fluoride added to the blood also produces incoagulability by precipitation of calcium, but the addition of soluble calcium salts does not make the mixture coagulable. Bordet and Gengou ${ }^{4}$ have shown that the precipitate of calcium fluoride thus formed had absorbed all or part of the coagulating ferments and even of the fibrinogen.

Now, if the amount of oxalate of sodium, used in the experiments described above, is slightly increased, results are obtained analogous to those got when fluoride is used. The insoluble oxalate of calcium has therefore operated like calcium fluoride to absorb some or all the substances (in the classic theory called the ferments) which give the blood its coagulating qualities. Even in smaller quantities the sodium oxalate possesses qualities noxious to the "coagulating forces" of the blood, though to a lesser degree. Citrate of soda is not open to these objections.

\section{The Action of Citrate of Sodium.}

Like oxalate and fluoride, citrate of sodium makes the blood incoagulable when added at the rate of about 1 per 1000 . The addition of a soluble calcium saltfor instance, calcium chloride-immediately restores the coagulating property. The citrate has produced incoagulability by acting on the calcium in the blood. But the difference in action of the citrate and of the oxalates and fluorides is shown directly by an easily repeated experiment. The addition of chloride of calcium to a solution of the latter causes a dense white precipitate. The addition of chloride of calcium to a solution of citrate of soda occasions no precipitate. Contrary, therefore, to the oxalate and fluoride, if the citrate acts on the calcium it does not do so by way of precipitation.

More than ten years ago this action of citrate of sodium on the calcium perplexed biologists (Sabattani,

2 Two other indirect methods with the same aim have been described: (a) Wright, Chantemesse, and Cathala take a series of drops of blood from the thumb and examine the quantity of the admixture of oxalate of potassium necessary to prevent coagula tion; (b) Et. Brissaud (Paris, Baillière, 1911) makes use of the properties of salted plasma, studied by Bordet and Gengou.

Arch. de Physiologie, 1890-1891.

4 Ann. de l'Inst. Pasteur, 1912.

Marcel Bloch: La Coagulabilité Sanguine, Thèse, Paris, 1914. 
1900-1901). In modern chemical terminology the calcium is in a latent state. In 1902 Arthus comes back to the question. ${ }^{6}$ By its immobilising action on the calcium salts of the blood the citrate prevents the transformation of the profibrin ferment into ferment. Similarly citrated milk does not coagulate under the influence of the lab-ferment. Arthus has endeavoured to determine the nature of the phenomenon by means of ingenious experiments.

Citrate of soda is shown to be antagonistic to precipitating salts, and it may be considered as endowed with direct antiprecipitative properties. Now, the alkali oxalates and fluorides possess, on the contrary no antiprecipitative action with regard to chloride of sodium. We have here a fundamental difference between the salts and the citrates.

We must add that Arthus's stable emulsions resemble colloidal solutions; that the alkaline earth salts and the calcium salts in particular have a very special precipitative action on them ; and that these same calcium salts have a fundamental part to play in the blood and its coagulation.

\section{The Action of the Citrate on the Blood Calcium.}

In 1902, at the end of his esisay, Arthus declared that it did not seem possible to set up any likely hypothesis about the anti-coagulating effect of the citrate in the blood. Contrary to Arthus, M. Luigi Sabattani ${ }^{7}$ thinks that the anti-coagulant action of the citrate can be explained. This is due to his conception of the state of the calcium in the blood. In order that coagulation may take place, not only must a certain quantity of calcium salts be present, but also the ionised state of the calcium. The citrate which does not precipitate the calcium salts leaves the quantity of dissolved calcium intact, which was in the blood and which was enough for its coagulation, but it modifies its state of ionisation and thus annihilates it functionally. ${ }^{89}$

\section{Part Played by Blood Calcium.}

When the blood is citrated the pre-existent calcium dissolved is in no way diminished in weight; the citrated blood contains as many molecules of calcium salts in solution as normal coagulating blood, and yet it does not coagulate. It will at once recoagulate if another calcium salt is added. It must therefore be admitted that the citrate has occasioned a change of state of the blood calcium which completely arrests its action in coagulation.

This phenomenon is not isolated, and modern chemists can readily furnish examples of what they call "bodies in a hidden state." Examples might be multiplied, but it is enough for us to know that a body-metal or metalloid-can exist in a solution in such a state that it is incapable of manifesting its properties. This is what the addition of the citrate has done in the blood with respect to the calcium. The citrate has hidden the calcium.

If we may borrow terms from modern serology without in any way comparing the phenomena, we might say that the citrate inactivates the calcium in a calcic solution -e.g., blood-and that the addition of a little more calcium suffices to reactivate the solution.

Let us now sum up some of the discoveries of modern physical chemistry. The bodies which may be added to a given colloidal solution behave in two ways. Some, the "electrolytes," tend to precipitate the solution-e.g., certain salts of the alkalis or alkaline earths; they are precipitants or instabilisers. Others reinforce the capacity of the solution to remain in the colloidal state; they are stabilisers.

Let us, then, look upon the blood plasma as a colloidal solution. From the physico-chemical point of view this is the modern conception; it is that nsed by Nolf in order to establish his theory of coagulation. This blood plasma contains electrolytic precipitants; they are the calcium salts. If certain bodies like citrate of

${ }^{6}$ Soc. de Biol., May 10th, 1902 (Inst. Pasteur de Lille). Soc. de Biologie, May 14th, 1902.
Arch. ital. de Biol., t. xxxri., f. 3 .

9 Phamac. expér. Cagliari. sodium are added to the blood they act as stabilisers of the colloidal blood solution and prevent the electrolytic calcium salts from exercising their peculiar precipitating properties, and this without in any way affecting the quantities of dissolved calcic salts. The ion calcium may therefore with the greatest ease be active and provoke coagulation, or inactive and bring about incoagulability.

\section{Suggested Hypothesis.}

For this reason we feel at liberty to set up the following hypothesis: The blood when fluid and circulating in the vessels is in analysis chemically identical with the coagulated blood in a glass tube. The forma tion of fibrin is due to a change of physical state of those constituent elements which circulate as fluids in the vessels. Now, if, as we have seen is the case, a simple change of physical state of the calcium is enough to cause or prevent the coagulation of the colloidal sanguine solution in vitro, why not suppose that in the blood circulating in vivo the calcium exists in the inactive latent state? May not simply the issuing from the vessels, exposure to the air, contact with the walls of the recipient and with Nolf's thromboplastic substances (dust, powders) be enough to trans. form this hidden inactive state of the calcium into an active electrolytic precipitant state with respect to the colloidal solution ? 10

It is possible that the mechanism of the activation of the calcium is not simple. The anti-coagulating (antithrombin) substances of the plasma are perhaps "stabilisers" of the colloidal blood solution, antagonistic to the calcic precipitating electrolytes. These are antiprecipitant bodies, which would change their physical state on egress from the vessels under the influence of exterior contacts, lose their stabilising power, and set free the action of the calcium salts.

In any case we are led to assign to the calcium salts of the plasma the main active part in the phenomenon of the fluidity of the blood and the act of coagulation. This part is summed up diagrammatically in the following table:-

$$
\text { Blood fluid in circulation. }
$$

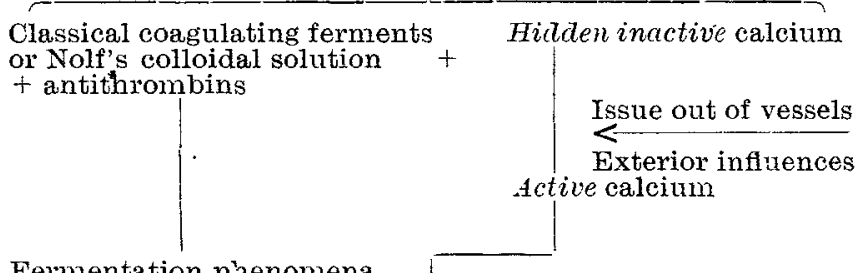

Fermentation p'enomena

or precipitation from the colloidal solntion

\section{Coagulation.}

Physico-chemical Conception of Coagulation and of the old "Diastasic Phenomona." Catalytic Coagulation Phenomenon.

There is in chemistry a whole category of phenomena produced only in presence of bodies, which do not seem to partake in the reactions because they are not modified, but nevertheless they are necessary to their production. This is what is termed catalysis. We believe that coagulation belongs to the category of these catalytic phenomena, calcium being the necessary active body, the "catalyser." 11

Nolf's idea, the theory of colloidal precipitations, is not as opposed as one might believe to the classical fermentation, enzyme, or diastase theories. Indeed, the modern theory of the diastases is very different

10 It is well known that certain writers of to-day assign great importance to the sudden changes caused in the physical equilibrium of the suaden changes caused in the physical blood blood colloids "by commonplace, purely physical, causes, such as cold" (Widal), To phenomena of this order would belong the blood crises (with coagulation disturbances), anaphylaxis, injections of heterogenous albumins, paroxysmal hæmoglobinuria, asthma, urticaria, \&c. See Widal, Abrami, E. Brisnaud, Joltrain Societe Medicale, Feb. Juth, 1914,

113; Presse Medicale, June, 1914. 11 This term has already been applied to coagulation. The fibrin ferment performs the transformation of the fibrinogen into fibri in the manner of a catalyser without 
from that held a few years ago. There is a tendency to draw nearer to the original opinion of Arthus and Naegeli, who denied that the action of the diastases was due to their chemical constitution, but considered that they acted like special transmissible forces, such as electricity and light.

We are acquainted with a large number of enzymic phenomena where the presence of a trace of an acid, alkali, or salt is of fundamental importance, like the trace of calcium salt in coagulation. The importance of other mineral substances (iron, manganese) is as great in other diastasic phenomena (G. Bertrand, Trillat, Phocas). Finally, the discovery of non-albuminoid diastases and of purely metallic colloidal solutions, having the property of diastases (Bredig, Trillat, Robin, Bardet), has completely modified our conception of these latter. Their catalytic action appears to be due, not to the nature of the metal itself, but to the colloidal form in which it is presented. Thus, in all these former fermentation phenomena the part played by the mineral element steps into the foreground. Consequently, the part in coagulation which we propose to assign to the calcium is not exceptional. As regards the intimate nature of the mineral element in the catalytic phenomenon, it is of necessity still entirely hypothetical.

Application to Method of Measuring Coagnlability.

In practice the blood is first mixed with a measured solution of citrate of sodium $(1 \mathrm{c.cm}$. of blood in $4 \mathrm{c.cm}$. of citrate of sodium of 1 to 400). The dilution and calcic inactivation prevent the intervention of foreign contacts, the cause of so many errors in direct coagula. tion observations.

The next step is to divide equal quantities $(2 / 10$ c.cm. $)$ of this citrated blood into a series of tubes containing increasing amounts of chloride of calcium (from $0.00005 \mathrm{~g}$. to $0.0005 \mathrm{~g}$. diluted in $4 \mathrm{c.cm}$. of isotonic salt solution).

At the end of several hours the series of tubes gives a succession of steps in coagulation, incomplete at first in the tubes containing little calcium, then more and more complete as we get near to the amount required for total coagulation. The tubes which mark commencement and end of coagulation are noted. The relationships between the quantities of citrate of sodium and chloride of calcium in these tubes constitute the "indices of coagulability." These indices are notably fixed for normal bloods (two at the beginning and one at the completion of coagulation); the stronger the coagulability the higher the indices, and inversely.

By'this procedure we have been able to reveal various experimental and pathological variations of coagulability which other methods did not permit us to appreciate. ${ }^{12}$ It allows of the most exact study of the qualities of the coagulum-normal retractility, hyper-or hypo-tractility, or irretractility.

Experimental Applications; Regulating Mechanism of Coagulability.

"In various organs (spleen, pancreas, lymphatic ganglions, small intestine, testes, brain, lungs) it is possible to reveal an anticoagulating substance, probably identical with the antithrombin which passes from the liver into the blood under the influence of the peptone; in the organs this substance is masked by one or more substances which energetically activate the coagulation of the blood in vitro" (Doyon).

These substances, multiglandular in origin and inverse in action, balance each other and their result is coagulability; it is easy to show the instability of this state of equilibrium. If an animal is bled quickly and freely, the succeeding hours show a sudden fall of the indices followed by a reactional elevation above the normal. A return to the normal takes place only after a certain time. (Fig. 1.) If an animal is bled by degrees a fairly durable state of hypercoagulability can be produced.

The same phenomenon may be shown in man, after sudden or repeated hæmorrhages. We have here, perhaps, the explanation of the hypercoagulability found in certain affections (uterine fibroma) and accidents (thromboses, post-operation embolism).

12 For details of procedure and results see Mrarcel Bloch, loc. cit.
Sudden loss of equilibrium may be observed on the sudden introduction into the animal of certain poisons, in particular of heterogeneous albumins. The experiment of the Schmitt-Müllheim type gives immediate prolonged

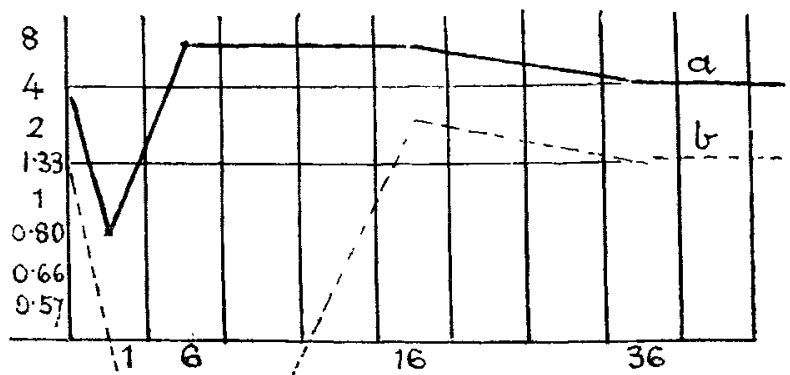

FIG. 1.-Fall followed by rise of coagulability after plentiful bleeding in the dog. Index of coagulability on the lett of the chart, time in

incoagulability of the blood of a dog which had just had an intravenous injection of propeptone with reactional hypercoagulability.

\section{Clinical Applications: Suaden Disturbances of Coagulability.}

We have here a new chapter of human pathology, which opened with the proof of disturbances of coagulability in the anaphylactic shock. They are com prehensible when we note the instantaneous formidable

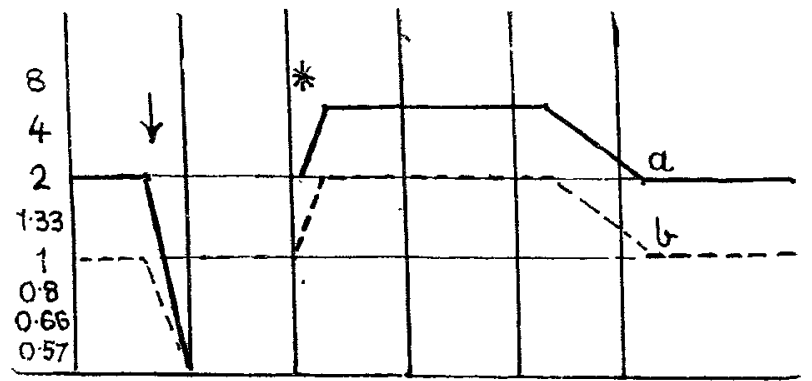

FIG. 2.-Incoagulability followed by reactional hypercoagulability after intravenous injection of peptone in the dog (shown by the arrow). The asterisk indicates a period 10 hours after the injection. $(a)=$ commencing, $(b)=$ complete, coagulation.

modifications undergone by the plasma elements in the experiment of peptone blood in the case of animals, and after copious hæmorrhages in men and animals.

(Fig. 2.)

In the course of experimental anaphylactic shocks (Richet, Riedl, Kraus) it is posisible to observe either the more or less complete incoagulability of the blood of the animal, or its hypercoagulability, which is often accompanied by fibrinolysis or redissolution of the clot. In human beings great anaphylactic shocks may be accompanied by phenomena of the hæmorrhagiferous class (purpura). ${ }^{13}$ Certain symptom-complexes, such as paroxysmal hæmoglobinuria, urticaria, asthma ${ }^{13}$ present coagulation disturbances in the course of the crisis (hypercoagulability, irretractility of the clot, fibrinolysis). In the fight against these hæmoclastic crises, or sudden entire losses of equilibrium of the plasma colloids, M. Widal has successfully used intravenous autoserotherapy.

Conversely it has been suggested to combat certain hæmorrhage conditions by means of the hypercoagulability consequent on anaphylactic shocks produced by reinjection of heterogenous serums (Dufour and Crow ${ }^{15}$ ).

$$
\text { Slow Disturbances of Coagulability. }
$$

These may be noted in numerous illnesses. With the index method we have determined the coagulability in a number of diseases.

13 J. Halle and Marcel Bloch: Choc anaphylactique dans la sérothérapie antidiphtérique (Soc. de Pédiatrie, February, 1913).

therapie antidiphterique (Soc. de Pediatrie, February, 1913). 1973 Wial, Abram, et Brissaud. Semaine Medicale, Dec. 24th, Feb 13th 1914, L'Urticaire Presse med, Id., et Joltrain, Soc. Méd., Feb. 13th, 1914, L Urticaire, Presse med., July 11th, 1914, L'Asthme. anaphylactique, Soc. méd. des hôpitaux, June 5th, 1914. 
With marked hepatic insufficiency affections of the liver produce a clear diminution of coagulability. The predominant part played by the liver amongst the organs regulating coagulability explains most of the hæmorrhagiferous accidents in liver cases.

Lesions of the kidney may also cause a lowering of the indices of coagulation, which partly explains the "hæmorrhagic diathesis" of certain cases of Bright's disease. In extensive codema the hydræmia seems to have a direct action in reducing coagulability.

Cardiac affections modify the indices in various ways, probably in accordance with the state of the organ or organs most severely attacked in their functions.

The lungs play a most important part in the secretion of coagulating substances (Doyon, Morel and Kareff, Roger $\left.{ }^{16}\right)$. Considered clinically a mechanical congestion of the lungs may produce considerable diminution of coagulability (Marcel Bloch ${ }^{17}$ ). The same observation can be made in ordinary pulmonary tuberculosis during the period of softening.

In simple pneumonia hypercoagulability is constantly present, persisting for several days after defervescence. It must be noted, from the point of view of diagnosis, that in influenza, typhoid, and miliary tubercle the indices are normal or lowered. For prognosis lowering of indices of coagulation is a sign of deterioration. It is found above all in pneumonia of alcoholic patients with fatal termination.

In polyglandular and endocrinous affections coagulability may be disturbed. P. E. Weil ${ }^{18}$ has recently insisted on the disturbances in coagulation in the course of menorrhagia of women presenting signs of ovarian, thyroid, pituitary, or suprarenal insufficiency.

In toxic infections coagulability is variable. Whilst always increased in acute articular rheumatism, it is usually lowered in typhoid fever, sometimes very greatly so in hæmorrhagic forms.

In certain toxic infectious cases, acute diphtheria, for instance, the indices are raised in spite of the hrmorrhagiferous conditions. Indeed, the key to the hæmor rhagiferous symptom complex is not always given by the study of coagulation; the conception of endotheliovascular insufficiency or dystrophy must be conjoined with it. It may be proved by the "signe du lacet" (Weill, of Lyons). The hypertension induced by light constriction of a portion of the member provokes the bursting of small vessels in the subjacent zone and the immediate appearance of a fine scattered purpura.

The study of the vascular resistance and of coagulability combined permits of interesting distinctions in the purpura and thrombosis symptom complexes. By the side of purpura with diminished coagulability there are purpuras with normal coagulability but irretrac tility of the clot (insufficiency of blood-plates) and purpuras with normal coagulability and diminution of vascular resistance (endothelio-vascular insufficiency or dystrophy).

Similarly, it is possible to distinguish venous thromboses with increased coagulability (aseptic thromboses in childbirth, coagulability indices increased at the end of pregnancy), distinct from infectious thrombophlebitis, and, finally, thromboses with endothelio vascular dystrophy, where the coagulability is norma but in which it is easy to prove a weakening of vascular resistance, sometimes hereditary in the family.

In the case of hemophilia the problem is still more complex. In true hæmophilia we have considerable lengthening of the coagulation period, abnormal coagulability indices, sometimes precocious start, but enormous decrease of index of complete coagulation. Hamophilia states must be placed beside them for comparison. These are states of very diminished coagulability and vascular resistance occurring in the severe types of purpura, hæmoglobinuria, pernicious anæmia, scurvy, \&c., and in the hæmorrhagic forms of the acute exanthems.

16 Roger, Les fonctions du youmon, Presse méd., November, 1912 17 Marcel Bloch: Loc. cit

${ }^{18}$ P. E. Weil : La durée des régles, les ménorrbagies, troubles de la coagulation sanguine, Soc. de Biol., April 27th, 1912; Traitement par le sérum sansuin, J. de Méd. int., Oct. 10th, 1913.
Previous Contributions.

The French Supplement to THE LANCET-under the Editorial Direction of Professor Charles Achard, Professor of Pathology and Therapeutics in the University of Paris; and Dr. Charles Flandin, D.S.O., MédecinMajor de 2me Classe, Chef de Clinique à la Faculté de Paris-has appeared on the dates given in the following list, which sets out the titles and authors of the contributions :-

Sept. 21st, 1918: (1) The Microbic Diversity of the Enteric Fevers, by Professor Achard. (2) Post-Typhoid Atony of the Cæcum, by Maurice Loeper, Professor of the Faculty of Medicine and Physician to the Paris Hospitals.

Oct. 19th, 1918: (1) What the War has Taught Us about Tetanus, by Louis Bazy. (2) Tetanus Consecntive to SuperficialWounds and to Trench Foot: Treatment and Prevention, by Professor Raymond, Médecin Prin cipal de 2me Classe.

Nov. 30th, 1918: (1) General Review of French Cardio. Pathology during the War, by Ch. Laubry, Physician to the Paris Hospitals, Médecin Major de 2me Classe. (2) Methods of Estimating Augmentation in Depth of Volume of Left Ventricle, by Dr. Bordet, Director of the Radiological Labo. ratory of the Centre of Cardiology of Professor Vaquez, Hôpital St. Antoine.

Jan. 11th, 1919: (1) Gunshot Concussion of the Spinal Cord, by Henri Claude, Professor in the Paris Faculty of Medicine, Physician to the Paris Hospitals; and Jean Lhermitte, formerly Chef de Clinique in the Paris Faculty of Medicine. (2) Contribution to the Study of the Manifes. tations of Emotional Shock on the Battlefield, by Cl. Vincent, Physician to the Paris Hospitals, formerly Medical Officer to the 46th and 98th R.I. and to the 44th B.C.P.

March 1st, 1919: The Surgical Complications following Exanthematic Typhus, by Dr. Paul Moure and Dr. Etienne Sorrel, Prosectors to the Faculty of Medicine, Paris, Medecins Aide-Majors de ler Classe, Surgeons to the French Hospital at Jassy.

April 5th, 1919: (1) Nervous Complications of Exanthematic Typhus, by A. Devaux, formerly Interne of the Paris Hospitals, Médecin-Major de $2 \mathrm{me}$ Classe. (2) Note on the Epidemic Diseases Observed in Rumania during the Campaign of 1916-17, by Dr. Henri Vuillet, late Interne of the Paris Hospitals.

May 3rd, 1919: (1) Malaria During the War, by G. Paisseau, Médecin-Major de $2 \mathrm{me}$ Classe. (2) Distribution of Soldiers, Temporarily Unfit through Malaria, in Agricultural Colonies, by Professor E. Jeanselme. (3) Studies on Renal Function in Chronic Nephritis, thesis by Pasteur Vallery-Radot, reviewed by Professor Charles Achard.

June 21st, 1919 : (1) Recent Work on Cerebro-spinal Fever, by C. Doptər, Professor, Val-de-Grâce, Médecin-Chef to an Infantry Division. (2) Meningococcal Rheumatism and Arthritis, by Dr. Paul Sainton, Physician to the Paris Hospitals.

Aug. 23rd, 1919: Radiological Examination of Pseudarthroses before and after Operations of Bony Grafts, by Paul Aubourg, Professor Agrégé, Cuneo's Laboratory for Radiology. (Centre for Surgery of Bones, Hospital 75, Cannes.)

Sept. 27th, 1919: (1) Dysidrosis: its Parasitic Nature, by J. Darier, Physician at the Hospital of St. Louis; Member of the Academy of Medicine. (2) The Contagiousness of Favus in Man, by $R$. Sabouraud.

Nov. 8th, 1919: (1) Secondary Echinococcus, by F. Dévé Professor at the School of Medicine, Rouen. (2) A Case of Peritoneal Echinococcus, by Charles Flandin, Chef de Clinique Médicale at the Faculty of Medicine in Paris.

Dec. 20th, 1919: French Work on Ictero-Hæmorrhagic Spirochætosis, by Marcel Garnier, Physician at the Lariboisière Hospital, Paris.

Jan. 31st, 1920: (1) Some Appliances for the Examination of the Nervous and Muscular Systems, by Dr. Jean Camus, Professeur Agrégé of the Medical Faculty in Paris; Médecin des Hôpitaux. (2) The Laws of Tremor, by Dr. Léon Binet, Interne des Hôpitaux, Paris.

THE LANCET can be ordered through any Library in France or hrough the following special agents :-

PARIS.-Masson Er Cie, 120, Boulevard St. Germain.

Emile Bougault, 48, Rue des Eeoles.

Ch. Boulaygti, 14, Rue de l'Anciénne Comédio.

Felix Alcan, 108, Boulevard St. Germain.

M. CholsNet, 30, Rue des St. Pères.

H. Le Souprer, 174, Boulevard St. Germaín.

Matorne et Fins, 27, Rue de l'Ecole de Médecine.

Vigot Frḱres, 23, Rue de l' Heole de Médecine.

MARSEILLES.-TACUSSEL AND Lombard, 54, Rue Paradis.

Toulouse.-Edouard Privat, 14, Rue des Arts. 


\section{THE LANCET.}

LONDON : SATURDAY, AUGUST 7, 1920.

\section{Unprincipled Medication.}

THE Proprietary Medicines Bill, introduced by Lord AsTOR in the House of Lords on July 13th, raises in particularly acute form the right of the State, or of one department of the State, to restrict the liberties of a flourishing industry on the plea of benefiting the community at large. No adversary of the measure could state the case in stronger terms than those which can be employed in its adrocacy. The Bill, now being discussed, sets out to probibit the sale of such remedies as are sold under a trade name or manufactured by a secret process, unless and until they have been registered by the Ministry of Health, a formality which assumes a disclosure in confidence of their composition. The Bill prohibits altogether, under penalty of heavy fine or imprisonment or both, the sale of remedies purporting to cure certain diseases or disorders, of which a preliminary list of 12 is given in the Schedule to the Bill. The list contains at present the following items: (1) cancer, (2) consumption, (3)' lupus, (4) deafness, (5) fits, (6) epilepsy, (7) amenorrhœa and other diseases peculiar to women, (8) diabetes, (9) paralysis, (10) locomotor ataxy, (11) Bright's disease, (12) rupture; and the Minister is to be empowered to extend, as he may think fit, by regulation laid before Parliament, a list already of a comprehensive nature. Other provisions in the Bill disallow the vendor of any proprietary remedy from entering into personal correspondence with those who buy and use his wares, and also the employment of the "trailer" advertisement-being the enclosure with one remedy of printed matter recommending another-becomes illegal. It is evident that if the Bill becomes law it will drastically restrict the operations of a trade forming a substantial section of the London Chamber of Commerce and known to contain more than 300 manufacturers or vendors.

Public opinion is, however, unanimous in support of any measure designed to prevent the sale of fraudulent medicines and of drugs or appliances intended for immoral purposes. All appear to be fully aware of the undesirability of "cure-all" claims. Yet considerable protest has been raised against the Proprietary Medicines Bill in certain journals, wherein it is variously described as an insincere measure designed for the glorification of the Ministry of Health, as a further invasion of public liberty under the pretext of coddling, and as a serious threat to the comfort and convenience of a large section of the public. It is even suggested that the victims of incurable cancer will be deprived by the Bill of the remedies required to soothe and alleviate their sufferings; that, in fine, it is not the public interest but rather the sectional interest of the medical profession which is at the bottom of the changes proposed. The history of the Bill easily disposes of it as a piece of sectional or self-interested legislation. The proposals are based upon and do not go beyond the recommenda. tions of the Select Committee appointed in June, 1914 , to inquire into the sale and advertisement of patent and proprietary medicines and appliances, and to report what amendment in the law was necessary. Doctors, as is natural, were members of that Committee, and doctors were heard in evidence; but in neither case had they any numerical predominance, and the report of the Committee, whose personnel was of a very varied character, was presented with unanimity. Medical men, indeed, did not themselves come out scatheless from an inquiry revealing almost incredible degrees of cupidity on the one side and gullibility on the other. The Committee spoke with regret of medical men who allowed themselves to be made "the catspaw and fool of the fraternity of the secret remedy," and they had evidence in two cases of medical men acting as directors of companies manufacturing secret remedies.

The plain truth is that the lurid evidence laid before the Select Committee, and quoted by Lord AsTor in support of the measure, was more than sufficient to move any body of citizens, not blinded by prejudice and self-interest, to immediate and forcible action. The sale of proprietary remedies had been increasing by leaps and bounds for some years before the Committee was appointed. Remedies prohibited in America were being shipped in enormous quantities for use in this country. Touting swindlers made fortunes here, only to meet with fine and imprisonment on attempting to do the same in Paris. Australia had refused to sanc. tion the importation of many of the remedies, and more of the literature, freely circulating in this country. Lastly, the attitude of the newspaper and periodical press in regard to secret remedies was, speaking generally, very lax. In 1914 advertisements of proprietary medicines constituted a considerable source of income for many journals ; a number of small provincial papers could hardly have existed at all without them, and the medical press was not too carefully censored. This fact led newspapers, either from discretion or under compulsion-we are now quoting from the report itself - to exclude from their columns criticism or discussion of secret remedies. For all these abuses the Select Committee thought that prompt remedies should be found, and upon their recommendations the new Bill has been framed for the protection of the public-and with that sole object.

\section{The Hospital Survey.}

Reference was made in a recent number of THE LANCET ${ }^{1}$ to Sir NAPIER BuRnetT's lucid and informing survey of the financial position of the voluntary hospitals throughout Great Britain and Ireland for the five years 1914-19. From this survey the statistics of the hospitals of London are excluded, as they come directly under the purview of King Edward's Hospital Fund, while there is no information as to 200 hospitals which left the request for information unanswered, and from 32 others which intimated that they had incurred no deficit, and therefore required no special assistance. We should have liked details as to how these desirable conditions were arrived at, while it is possible that some of the 200 who remained silent were similarly fortunate. It is to

1 THE LAANCET, July $20 t \mathrm{th}, \mathrm{p} .83$. 